Rev. Adm. Saúde - Vol. 18, № 70, jan. - mar. 2018

http://dx.doi.org/10.23973/ras.70.70

ARTIGO ORIGINAL

\title{
Adesão à identificação do paciente em hospital universitário público
}

Adherence to patient identification in a public university hospital

\begin{abstract}
Akie Fujii Neta1 ${ }^{1}$, Camila Girardi' ${ }^{2}$, Daiana Tais Rheinheimer dos Santos ${ }^{3}$, João Lucas Campos de Oliveira ${ }^{4}$, Renata Pereira de Oliveira ${ }^{5}$, Maristela Salete Maraschin $^{6}$, Nelsi Salete Tonini ${ }^{7}$
\end{abstract}

1. Graduanda em enfermagem da Universidade Estadual do Oeste do Paraná (UNIOESTE).

Cascavel, PR

2. Enfermeira, pós-graduanda no Programa de Residência em Gerenciamento de Enfermagem em Clínica Médica e Cirúrgica da Universidade Estadual do Oeste do Paraná (UNIOESTE). Cascavel, PR

3. Enfermeira, pós-graduanda no Programa de Residência em Gerenciamento de Enfermagem em Clínica Médica e Cirúrgica da Universidade Estadual do Oeste do Paraná (UNIOESTE). Cascavel, PR

4. Enfermeiro, doutor em enfermagem. Docente colaborador da graduação em enfermagem e do Programa de Residência em Gerenciamento de Enfermagem em Clínica Médica e Cirúrgica da Universidade Estadual do Oeste do Paraná (UNIOESTE). Cascavel, PR

5. Enfermeira, especialista em saúde pública. Coordenadora do Serviço de Gestão da Qualidade do Hospital Universitário do Oeste do Paraná (HUOP). Cascavel, PR

6. Enfermeira, mestre em enfermagem. Professora adjunta ao Colegiado de Enfermagem da Universidade Estadual do Oeste do Paraná (UNIOESTE). Cascavel, PR

7. Enfermeira, doutora em enfermagem psiquiátrica. Professora associada ao Colegiado de Enfermagem da Universidade Estadual do Oeste do Paraná (UNIOESTE). Cascavel, PR

\section{RESUMO}

Estudo transversal cujo objetivo foi identificar a taxa de adesão à identificação do paciente hospitalizado, bem como os principais motivos para sua não aderência. $A$ 
pesquisa foi realizada em hospital universitário público do Paraná, com uma amostra $(n=748)$ de pacientes internados entre setembro a dezembro de 2016. A coleta de dados foi precedida por observação direta com registro da adesão à pulseira de identificação e motivos para não aderência em um dia aleatório não agendado de cada mês. Aos dados tabulados procedeu-se análise estatística descritiva em medidas de proporção. A taxa geral de adesão à identificação do paciente foi de $71,6 \%$. O melhor resultado de adesão foi constatado no centro obstétrico (94,2\%); e o pior, na pediatria, com $61,4 \%$ de não adesão. O motivo de não aderência mais frequente se relaciona à perda da pulseira nos setores.

Concluiu-se que a adesão à identificação do paciente no hospital ainda é deficitária, e que a melhor comunicação com os setores assistenciais e o serviço de internamento pode ser uma estratégia para a maior adesão.

Palavras-chave: sistemas de identificação de pacientes, segurança do paciente, indicadores de qualidade em assistência à saúde, gestão da qualidade, enfermagem.

\section{ABSTRACT}

A study carried out with the objective of identifying the rate of adherence to the identification of hospitalized patients, and the main reasons for not using. The study was developed in a public university hospital in Paraná, with a sample $(n=748)$ of hospitalized patients between September and December 2016. The data collection was performed once a month on a day not scheduled for observation and registration of the use of the identification bracelet and the reasons for not accepting it. A descriptive statistical analysis of the data obtained was performed in proportion measures. The overall adhesion rate to patient identification was $71.6 \%$. The best utilization result was found in the obstetric center (94.2\%); and the worst, in pediatrics, with $61.4 \%$ of non-use. The main reason for not wearing the identification bracelet was pointed out by the loss in the sectors. It was concluded that adherence to patient identification at the hospital is still deficient, better communication with the care sectors and the inpatient service may be a strategy to increase the use of the bracelet.

Keywords: patient identification systems, patient safety, indicators of quality in health care, quality management, nursing.

\section{INTRODUÇÃO}

A segurança do paciente deve ser objeto de preocupação em todas as esferas de gestão e assistência na saúde, tornando-se proposta indissociável para o almejo da qualidade do cuidado, uma vez que os riscos associados à sua produção são evidentes. Assim, no Brasil, a portaria nํ529 de 1ำ de abril de 2013 institui o 
Programa Nacional de Segurança do Paciente, que tem como objetivo geral contribuir para a qualificação do cuidado em todos os estabelecimentos de saúde do território nacional ${ }^{(1)}$.

Buscar a qualificação do cuidado e alavancar a segurança na assistência à saúde é um desafio que precisa ser continuamente (re)planejado. Para isso, ações sistemáticas e estratégicas são necessárias à viabilização da racionalidade desta busca ${ }^{(2)}$. Logo, o manejo da qualidade e da segurança da paciente se baseia em princípios de disseminação da cultura organizacional em prol do cuidado seguro (3-4); do uso de ferramentas sistemáticas para a melhoria assistencial e redução de riscos ${ }^{(3,5)}$; da educação permanente de profissionais ${ }^{(6)}$; e também, inclusive, da maior participação do paciente durante a prestação da assistência e conhecimento sobre esta, que ele consome ${ }^{(7)}$.

Em suma, quando a gestão da qualidade e da segurança do paciente se fundem, as ações estratégicas se baseiam no monitoramento de práticas de interesse ao cuidado qualificado e seguro; viabilização de ciclos de melhoria e

(re)replanejamento da melhor qualidade e segurança ${ }^{(8)}$. No contexto das melhorias amplamente recomentadas para a segurança do paciente, as ações devem se basear em estratégias racionais, tais como: identificar o paciente corretamente; melhorar a comunicação entre os profissionais de saúde; melhorar a segurança na prescrição, no uso e na administração de medicamentos; assegurar cirurgia em local de intervenção, procedimento e pacientes corretos; higienizar as mãos para evitar infecções; e reduzir o risco de quedas e lesões por pressão ${ }^{(3,8)}$.

A identificação do paciente é uma ação importante e primária na busca pela segurança na assistência, sendo inclusive no Brasil, solidificada por meio de protocolo norteador para ação ${ }^{(3)}$. Esta é uma ação simples que tem o objetivo central de garantir que o cuidado planejado seja prestado ao paciente proponente, ou seja, o certo ${ }^{(3,9-10)}$. Portanto, a identificação do paciente tem potencial para reduzir erros e eventos adversos que podem ser desastrosos à saúde ou até mesmo da vida dos consumidores da assistência ${ }^{(11)}$.

Atualmente, a recomendação geral da Agência Nacional de Vigilância Sanitária (ANVISA) no protocolo de identificação do paciente se dá pelas seguintes intervenções: identificar corretamente o paciente; educar o paciente/familiar para seu envolvimento no cuidado; e confirmar a identificação dos pacientes antes de proceder o cuidado ${ }^{(3)}$. A identificação de todos os pacientes (internados, em regime de hospital dia, ou atendidos no serviço de emergência ou no ambulatório) deve ser realizada em sua admissão no serviço através de uma pulseira. Essa intervenção deve permanecer durante todo o tempo que paciente estiver submetido ao cuidado (3).

A pulseira de identificação do paciente deve conter, pelo menos, dois identificadores, tais como: nome completo do paciente; nome completo da mãe (para recém-nascidos); data de nascimento; número do prontuário, etc. A pulseira identificadora deve ser branca, impermeável e passível de identificar o paciente manual ou digitalmente, por meio impresso. O protocolo normatiza claramente algumas especificações como necessidade de rodízio de membros; não utilizar pulseiras coloridas para finalidade de identificação, entre outros passíveis de consulta (3). 
Considerando a importância da identificação do paciente como estratégia para a viabilização do cuidado mais seguro; de que a ação já é normatizada oficialmente no Brasil, com possibilidade de adaptação às realidades organizacionais ${ }^{(3)}$ e que a avaliação é a égide da gestão comprometida com a qualidade e segurança na saúde (11), postula-se que os estudos que identifiquem a adesão à tal medida são elementares na assertividade de tomada de decisão rumo a melhorias. Ademais, divulgações científicas na problemática enunciada tendem a difundir o tema, e, assim, aumentar a visibilidade da identificação do paciente nos espaços assistenciais.

Posta a justificativa para se dedicar à pesquisa no problema enunciado, emergiram as seguintes indagações: Qual é a taxa de adesão à identificação do paciente hospitalizado? Quais são os motivos de não adesão à identificação do paciente? Para tanto, este estudo objetivou identificar a taxa de adesão à identificação do paciente hospitalizado, bem como os principais motivos para sua não aderência.

\section{MÉTODOS}

Estudo transversal, observacional, descritivo, e de abordagem quantitativa. Foi realizado em um hospital universitário público que atende à demanda exclusiva do Sistema Único de Saúde (SUS) com capacidade operacional de 215 leitos. A organização situa-se no interior do estado do Paraná, Brasil, e é referência para o atendimento ao trauma; neurocirurgia; gestação de alto risco e tratamento do HIV para uma população de aproximadamente dois milhões de habitantes.

A população de estudo compreendeu os pacientes de todos os serviços assistenciais de regime hospitalar (internação) nos meses de setembro a dezembro de 2016, a saber: clínica médica e cirúrgica geral e cardiologia; neurologia, ortopedia e vascular; maternidade (que foi dividida na observação pelas mães e recém-nascidos); centro obstétrico; psiquiatria; pronto-socorro (que foi dividido na observação pela sala de emergência que aloja pacientes e leitos de internamento); pediatria; unidade de terapia intensiva adulto; unidade de terapia intensiva pediátrica; unidade de terapia intensiva neonatal e unidade de cuidados intermediários.

A coleta de dados aconteceu em um dia aleatório de cada um dos quatro meses de análise, sem agendamento prévio. Não houve amostragem, pois, todos os pacientes foram previamente considerados como elegíveis. $O$ único critério de exclusão para participação foi a impossibilidade de o paciente responder por si mesmo, sendo menor do que 18 anos de idade e/ou com condição clínica desfavorável à resposta consciente na ausência de um familiar ou responsável. Assim, nas unidades de terapia intensiva (uti), sala de emergência e unidade de cuidados intermediários, a coleta aconteceu nos períodos de visita. A coleta de dados foi procedida por uma única pesquisadora, graduanda em enfermagem, previamente treinada pelas enfermeiras do Serviço de Gestão da Qualidade e Núcleo de Segurança do Paciente (NSP).

Para a coleta de dados, utilizou-se um formulário para computação manual dos dados dicotômicos de conformidade (adesão) ou inconformidade (não adesão) à 
pulseira de identificação do paciente, por setor hospitalar. Na instituição pesquisada, a pulseira é padronizada em cor branca, em material impermeável, e os identificadores nome completo (ou nome da mãe para recém-nascidos), data de nascimento e número de atendimento são empregados de forma impressa. 0 posicionamento da pulseira deve ser feito pelo setor de internamento. A instituição deste protocolo se deu formalmente no hospital em julho de 2014, promovida pelo NSP.

Em caso de não adesão à pulseira identificadora do paciente, a pesquisa avançou ao conhecimento do motivo para tal inconformidade. Assim, esta variável (não adesão) foi posteriormente desmembrada em: não aderida pelo internamento; perdida no setor e não reposta; e, não informado, quando o paciente ou responsável não soube relatar a razão para a ausência da pulseira identificadora. Optou-se por não investigar os motivos de não adesão à identificação das pacientes no centro obstétrico do hospital, pois o setor estava em reforma, e esta etapa da coleta demandava mais tempo, o que atribulava o trabalho da equipe. Contudo, a adesão/não adesão à pulseira de identificação foi observada neste serviço normalmente.

Todos os dados coletados foram transportados para planilhas eletrônicas do software Microsoft Office Excel, versão 2010. Aos dados tabulados, procedeu-se a análise estatística descritiva, com uso de medidas de proporção, conforme, inclusive, recomenda o protocolo de identificação do paciente da ANVISA na descrição do seu monitoramento por indicador de adesão ${ }^{(3)}$.

Ressalta-se que todas as exigências éticas que regem as pesquisas com seres humanos foram respeitadas. Acerca disso, o estudo faz parte de um projeto de pesquisa mais amplo, que entorna a avaliação da qualidade hospitalar por meio de indicadores assistenciais e gerenciais, que fora aprovado por comitê de ética institucionalizado, recebendo parecer favorável de protocolo $\mathrm{n}^{\circ}$ 1.696.925/2016 e CAAE: 58636916.5 .0000 .0107

\section{RESULTADOS}

Ao todo foram observados 748 pacientes. Destes, 535 estavam identificados. A taxa geral de adesão à identificação do paciente foi de $71,6 \%$, portanto, com $28,4 \%$ de não adesão. A Tabela 1 apresenta os resultados de adesão e não adesão à identificação do paciente por setor hospitalar.

Tabela 1. Adesão e não adesão à pulseira de identificação do paciente, por setor de atendimento hospitalar.

\begin{tabular}{|c|c|c|c|c|c|c|}
\hline \multirow[t]{2}{*}{ Setor } & \multicolumn{2}{|l|}{ Adesão } & \multicolumn{2}{|l|}{ Não Adesão } & \multicolumn{2}{|c|}{ otal } \\
\hline & $\mathbf{n}$ & $\%$ & $\mathbf{n}$ & $\%$ & $\mathbf{n}$ & $\%$ \\
\hline $\begin{array}{l}\text { Clínica médica e cirúrgica geral e } \\
\text { cardiologia }\end{array}$ & 60 & 63,9 & 34 & 36,1 & 94 & 100 \\
\hline
\end{tabular}




\begin{tabular}{lrrrrrr} 
Neurologia, ortopedia e vascular & 52 & 62,7 & 31 & 37,3 & 83 & 100 \\
Maternidade (mãe) & 83 & 91,2 & 8 & 8,8 & 91 & 100 \\
Maternidade (recém-nascido) & 75 & 90,3 & 8 & 9,7 & 83 & 100 \\
Centro obstétrico & 33 & 94,2 & 2 & 5,8 & 35 & 100 \\
Psiquiatria & 17 & 60,8 & 11 & 39,2 & 28 & 100 \\
Pronto-socorro (emergência) & 11 & 64,8 & 6 & 35,2 & 17 & 100 \\
Pronto-socorro (internação) & 84 & 85,8 & 14 & 14,2 & 98 & 100 \\
Pediatria & 27 & 38,6 & 43 & 61,4 & 70 & 100 \\
Unidade de cuidados intermediários & 27 & 65,9 & 14 & 34,1 & 41 & 100 \\
Unidade de terapia intensiva adulto & 27 & 49,0 & 28 & 51,0 & 55 & 100 \\
Unidade de terapia intensiva & 8 & 53,3 & 7 & 46,7 & 15 & 100 \\
pediátrica & & & & & & \\
Unidade de terapia intensiva & 31 & 81,6 & 7 & 18,4 & 38 & 100 \\
neonatal & & & & & & \\
\hline
\end{tabular}

A Tabela 2 sumariza os achados de interesse às razões para não identificação do paciente internado nos setores de atenção à saúde do adulto.

Tabela 2. Frequência dos motivos para não adesão à pulseira de identificação do paciente, por setor de atendimento hospitalar para adultos.

\begin{tabular}{llrr}
\hline Setor & Motivos & $\mathbf{n}$ & $\%$ \\
\hline Clínica médica e cirúrgica geral e & & & 53,0 \\
cardiologia & Internamento & 18 & 44,0 \\
& Perdida no setor & 15 & 3,0 \\
& Não informado & 1 & $\mathbf{1 0 0}$ \\
Neurologia, ortopedia e vascular & Total & $\mathbf{3 4}$ & 61,3 \\
& Internamento & 19 & 29,0 \\
& Perdida do setor & 9 & 9,7 \\
Unidade de terapia intensiva adulto & Não informado & 3 & $\mathbf{1 0 0}$ \\
& Total & $\mathbf{3 1}$ & - \\
& Internamento & - & 85,8 \\
& Perdida no setor & 24 & 14,2 \\
Pronto-socorro (emergência) & Não informado & 4 & $\mathbf{1 0 0}$ \\
& Total & $\mathbf{2 8}$ & 100 \\
& Internamento & 6 & - \\
& Perdida no setor & - & $\mathbf{1 0 0}$
\end{tabular}




\begin{tabular}{llrr}
\hline Pronto-socorro (internamento) & Internamento & 10 & $\mathbf{7 1 , 4}$ \\
& Perdida no setor & 2 & 14,3 \\
& Não informado & 2 & 14,3 \\
& Total & $\mathbf{1 4}$ & $\mathbf{1 0 0}$ \\
Psiquiatria & Internamento & - & - \\
& Perdida no setor & 11 & 100 \\
& Não informado & - & - \\
& Total & $\mathbf{1 1}$ & $\mathbf{1 0 0}$ \\
\hline
\end{tabular}

Por fim, a Tabela 3 também ilustra os achados atinentes aos motivos de inconformidade na adesão à identificação do paciente, contudo, entre os setores de atendimento materno-infantil.

Tabela 3. Frequência dos motivos para não adesão à pulseira de identificação do paciente, por setor de atendimento hospitalar materno-infantil.

\begin{tabular}{llrr}
\hline Setor & Motivos & $\mathbf{n}$ & $\%$ \\
\hline Maternidade (mãe) & Internamento & 4 & 50,0 \\
& Perdida no setor & 4 & 50,0 \\
& Não informado & - & - \\
& Total & $\mathbf{8}$ & $\mathbf{1 0 0}$ \\
Maternidade (recém-nascido) & Internamento & 3 & 37,5 \\
& Perdida do setor & 5 & 62,5 \\
& Não informado & - & - \\
Pediatria & Total & $\mathbf{8}$ & $\mathbf{1 0 0}$ \\
& Internamento & 7 & 16,2 \\
& Perdida no setor & 36 & 83,8 \\
& Não informado & - & - \\
Unidade de terapia intensiva pediátrica & Total & $\mathbf{4 3}$ & $\mathbf{1 0 0}$ \\
& Internamento & 1 & 14,2 \\
& Perdida no setor & 6 & 85,8 \\
& Não informado & - & - \\
Unidade de cuidados intermediários & Total & $\mathbf{7}$ & $\mathbf{1 0 0}$ \\
& Internamento & 1 & 14,2 \\
& Perdida no setor & 2 & 28,6 \\
& Não informado & 4 & 57,2 \\
& Internal & $\mathbf{7}$ & $\mathbf{1 0 0}$ \\
& Perdida no setor & 1 & 7,1 \\
& Não informado & 3 & 21,4
\end{tabular}




\section{DISCUSSÃO}

A identificação do paciente vem sendo debatida ao longo dos últimos anos, sendo uma das principais estratégias escolhidas pelo Ministério da Saúde para minimizar os eventos adversos relacionados à inversão de informações pessoais relacionadas a cada indivíduo internado em uma instituição de saúde ${ }^{(1,3)}$. O Ministério da Saúde exige que cinco compromissos (identificação do paciente, melhor comunicação entre os profissionais, melhor segurança na prescrição e administração de medicamentos, assegurar cirurgia em local de intervenção/ procedimento e paciente correto, higienização das mãos para evitar infecções e redução de risco de quedas e lesão por pressão) sejam firmados para que o básico de segurança e qualidade na assistência aconteça ${ }^{(1)}$. Acerca disso, considerando a relevância da identificação do paciente e a sua característica primária na busca pelo cuidado seguro, sendo discutida com maior ênfase já a partir de 2013 no Brasil, considera-se que a taxa geral de identificação do paciente no hospital de inquérito foi deficitária.

O hospital em estudo, desde o ano de 2016, utiliza-se de estratégias desenvolvidas pelo Núcleo de Segurança do Paciente e Gestão da Qualidade (NSPGQ) para que ocorra a melhora na proporção de adesão ao uso da pulseira de identificação, sendo esta incluída como um item de verificação das ações do Serviço de Infecção Hospitalar, quando realiza a busca ativa de casos. Assim, verifica-se se o paciente está ou não identificado, bem como na visita diária realizada pelos enfermeiros assistenciais para detectar o uso da pulseira, como também é recomendado que sejam feitas orientações para os pacientes e familiares quanto à importância da identificação. Portanto, os dados da pesquisa servem para fortalecer a tomada de decisão do NSPGQ no sentido de intervir mais incisivamente nos setores cuja taxa de adesão é claramente baixa, como o caso da UTI para adultos e, principalmente, da Pediatria.

O pior resultado de adesão à identificação do paciente considerado na unidade de pediatria é um dado alarmante e que merece total atenção das lideranças do serviço. Isso porque, ainda que a identificação do paciente deva ser um bem disseminado a todas as esferas organizacionais, sabe-se que, clinicamente, as crianças podem ser desestabilizadas mais rápida e gravemente do que um adulto em caso de um erro. Logo, o resultado de mais de $60 \%$ de falha na identificação dos pacientes internados neste setor é um fato que precisa ser revisto com urgência.

Com relação ao número geral de pacientes não identificados, pode-se considerar como elevada não conformidade, totalizando mais de um quarto dos pacientes do estudo. Não se tem conhecimento sobre o número aceitável de não conformidade, porém, acompanhar a proporção de pacientes utilizando a pulseira padronizada é uma das práticas recomentadas no protocolo de identificação do paciente, visando a sua melhoria contínua ${ }^{(3)}$. Considera-se a proporção de não adesão à pulseira identificadora como um resultado aquém do esperado, visto que o percentual de pacientes identificados deveria aproximar-se dos $100 \%$, por se tratar de uma etapa importante que, quando usada racionalmente, deve anteceder os cuidados. Reforça 
essa alusão o fato de que em outro estudo, os autores recomendam que a taxa de erros relativos a pulseiras de identificação deva ser mantida entre $0,2 \%$ e $0,3 \%{ }^{(12)}$.

Em estudo realizado na Europa por meio de uma auditoria realizada em 89 hospitais, evidenciou-se que a identificação do paciente era pouco executada, com índices baixos de conformidades relacionadas à identificação do paciente adulto (13). Assim, percebe-se que a identificação do paciente pode não ser um problema exclusivo do serviço pesquisado, já que isso demanda a cultura organizacional em prol maciço deste bem. Para que a instituição estabeleça suas metas de melhoria contínua, postula-se que a utilização de comparações com resultados internos e externos é uma estratégia profícua.

Em contraponto aos achados de teor negativo, os resultados constatados no setor de maternidade, tanto com relação ao atendimento da mulher como do recémnascido podem ser considerados satisfatórios, à comparação interna, devido às taxas de conformidade acima de $90 \%$, assim como no centro obstétrico do hospital, que representou o melhor setor na observação de aderência à identificação do paciente. Isso demonstra que há uma preocupação maior nessas unidades para identificação do paciente. Corroborando com esses dados, estudo realizado com objetivo de avaliar a identificação do paciente em unidades obstétricas, apontou que houve conformidade relacionada ao uso da pulseira por puérperas em $87,2 \%{ }^{(14)}$.

Destaca-se que o motivo "pulseira perdida no setor" foi o mais expressivo, considerando situações como rompimento da integridade da pulseira no setor de observação seguido da não reposição e/ou retirada da pulseira por algum profissional do setor. Sabe-se que adotar práticas aprimoradas e novas rotinas de trabalho nas instituições de saúde é um processo complexo, devido ao tempo de adaptação das equipes a políticas inovadoras. As rotinas já estabelecidas asseguram uma zona de conforto aos profissionais, enquanto a proposta de reconfiguração das ações pode desencadear sentimento de insegurança, rejeição e medo. Neste aspecto, a difícil tarefa de implantar novas rotinas foi descrita em estudo anterior, reconhecendo que políticas que visam alterar o comportamento dos praticantes na melhoraria da segurança do paciente têm menos possibilidade de sucesso se não levarem em conta as práticas já existentes (15).

Ante o exposto, fica claro que as pulseiras identificadoras perdidas no setor são pouco repostas. Dito isso, a pesquisa serve como alicerce para a instituição e também para hospitais que vivenciem a mesma situação no sentido de reforçar a melhor comunicação dos setores de internação com os serviços responsáveis à provisão da pulseira identificadora, quando no caso da perda da mesma.

Os dados relacionados à identificação do paciente na UTI para adultos demonstraram um dos maiores percentuais de não conformidade (51\%), sendo destes $85,8 \%$ foram de pulseiras perdidas no setor. São várias "justificativas" que podem ser elencadas para o achado nesta unidade, tais como: a identificação do paciente neste setor permanece fixada no leito de internação, o serviço de internamento hospitalar não adentra as instalações da unidade para que pulseira seja posta corretamente e/ou muitas vezes as informações constadas no prontuário do cliente não são fidedignas. Outro motivo empiricamente conhecido é que os profissionais da UTI alegam que a pulseira identificadora prejudica a circulação sanguínea do paciente que não raras vezes se apresenta edemaciado. 
O processo de retenção de líquidos e formação de edema também expõe o paciente a riscos, devido ao quadro clínico e às terapêuticas empregadas, sendo necessária a realização de inspeções rotineiras pela equipe de assistência para se evitar o garroteamento do membro com a pulseira identificadora ${ }^{(16)}$. Apesar disso, a recomendação da identificação do paciente é normatizada por meio de pulseira (3), já que o uso de placas identificadoras pode não ser seguro tão quanto à pulseira, uma vez que na saída do paciente da UTI a placa identificadora pode permanecer com a entrada de outro doente internado.

Reforça a alusão expressa, estudo realizado em UTI que apresentou resultados divergentes da pesquisa ora descrita. Foram realizadas 400 observações relacionadas com o uso de pulseiras de identificação pelos pacientes críticos. Em 385 (96\%) dessas, os pacientes estavam identificados com a pulseira. Em 21, $(5,45 \%)$ das observações, a pulseira não estava colocada adequadamente no paciente, dificultando a visualização destas pelos profissionais ${ }^{(16)}$. Em outras palavras, aliando os resultados deste estudo com a literatura correlata, é possível identificar que a taxa de adesão à identificação do paciente na UTI do hospital de inquérito foi, de fato, deficitária.

Concernente aos dados da unidade de terapia intensiva neonatal, a qual apresentou $81,6 \%$ de adesão à pulseira identificadora, há estudo com resultados similares, sendo este realizado em uma UTI neonatal do Rio de Janeiro, que constatou que $77,5 \%$ dos neonatos apresentavam pulseiras de identificação ${ }^{(17)}$. A mesma pesquisa citada refere que uma possibilidade de justificativa para a não adesão em alguns casos ao uso da pulseira de identificação pode ser o fato de alguns neonatos permanecerem por muito tempo internados, o que gera nos profissionais de enfermagem, em especial, a sensação do conhecer o paciente ${ }^{(17)}$, o que sem dúvida é preocupante, pois a falha humana é inerente ao trabalho em saúde. Isso porque, a ausência de identificação do paciente pode promover eventos adversos graves, tais como erros na realização de procedimentos, na administração de medicamentos ou hemoderivados e até mesmo a troca de bebês ${ }^{(17)}$.

As não conformidades decorrentes do processo de identificação do paciente podem implicar em sérios eventos adversos, constituindo-se como um fator preocupante da assistência à saúde ${ }^{(14)}$. Em pesquisa realizada em uma maternidade do nordeste do Brasil, no ano de 2012, revelou-se que $76 \%$ dos casos de eventos adversos resultaram em de danos temporários e/ou prolongamento do tempo de internação hospitalar, bem como, apontou-se ainda que $87 \%$ dos eventos adversos estudados eram preveníeis ${ }^{(18)}$.

Em se tratando de erros relacionados à identificação do paciente, outra pesquisa realizada no estado de São Paulo analisou 74 erros relacionados a medicamentos em uma unidade de clínica médica, demonstrando que 2,7\% dos erros de administração de medicamentos ocorreram devido a não checagem da identificação do paciente antes da administração do fármaco ${ }^{(19)}$. Isso reforça, certamente, que a identificação do paciente é uma etapa primária na segurança assistencial, e que o seu uso racional pode ser uma barreira simples, porém eficiente, de falhas plenamente evitáveis. 


\section{CONCLUSÃO}

Conclui-se que a adesão à identificação do paciente no hospital ainda era deficitária, em especial considerando a instituição do protocolo relacionado em torno de dois anos antes da pesquisa. Alguns setores apresentam taxas mais satisfatórias de adesão à pulseira identificadora na comparação interna, como maternidade e centro obstétrico, o que reforça que esta é uma prática que demanda compromisso organizacional sistêmico, este, produto da prática individual consciente.

O motivo mais frequente de perda da pulseira identificadora no setor assistencial sinaliza que a comunicação merece ser uma estratégia continuamente estimulada, pois tão logo identificada a ausência da pulseira, os setores assistenciais precisam agir para que esta seja posicionada no paciente. Todavia, o papel do setor de internamento também deve ser incessantemente reforçado à sua importância, pois o ideal é o paciente ser identificado assim que preliminarmente atendido.

A impossibilidade de generalização de resultados e a ausência de emprego de estatística analítica/inferencial são limitações a serem expressas ao estudo. Porém, acredita-se que a pesquisa contribui solidamente à militância pela segurança do paciente, uma vez que a sua identificação é um passo primário e elementar na redução de erros e eventos adversos. Assim, o estudo difunde a temática e a possibilidade de que os resultados sejam comparados por outras localidades, o que facilita o estabelecimento de metas para a melhoria contínua no que se refere à adesão à identificação do paciente.

\section{AGRADECIMENTOS}

À Fundação Araucária pela concessão de bolsa de iniciação científica.

\section{REFERÊNCIAS}

1. Brasil. Ministério da Saúde. Portaria no 529, de 1ㅇde abril de 2013. Institui o Programa Nacional de Segurança do Paciente (PNSP). 2013. http://bvsms.saude.gov.br/bvs/saudelegis/gm/2013/prt0529_01_04_2013.html

2. Reis GAX, Hayakawa LY, Murassaki ACY, Matsuda LM, Gabriel CS, Oliveira MLF. Nurse manager perceptions of patient safety strategy Implementation. Texto Contexto Enferm, 2017. 26(2):e00340016. DOI: http://dx.doi.org/10.1590/0104-07072017000340016.

3. Ministério da Saúde. Anvisa. Protocolo de identificação do Paciente. Protocolo integrante do Programa Nacional de Segurança do paciente. 2013. Disponível em: http://bvsms.saude.gov.br/bvs/publicacoes/documento_referencia_programa_nac ional_seguranca.pdf 
4. Alves DFS, Guirardello EB. Nursing work environment, patient safety and quality of care in pediatric hospital. Rev Gaúcha Enferm. 2016. 37(2):e58817. DOI: http://dx.doi.org/10.1590/1983.

5. Oliveira RM, Leitão IMTA, Silva LMS, Figueiredo SV, Sampaio RL, Gondim MM. Estratégias para promover segurança do paciente: da identificação dos riscos às práticas baseadas em evidencias. Escola Anna Nery Revista de Enfermagem. 2014. 18(1):122-129. DOI: http://dx.doi.org/10.5935/1414-8145.20140018

6. Wegner W, Silva SC, Kantorski KJC, Predebon CM, Sanches MO, Pedro ENR. Educação para cultura da segurança do paciente: Implicações para a formação profissional. Escola Anna Nery. 2016. 20(3):e20160068. DOI: 10.5935/14148145.20160068

7. Pedro DRC, Silva GKT, Molin TD, Oliveira JLC, Nicola AL; Nelsi Salete Tonini, NS. Conhecimento do paciente sobre a assistência hospitalar recebida durante sua internação. Rev. REME, 2016. 20:e978. DOI: http://www.dx.doi.org/10.5935/1415-2762.20160048.

8. Ministério da Saúde. Documento de referência para o Programa Nacional de Segurança do Paciente / Ministério da Saúde; Fundação Oswaldo Cruz; Agência Nacional de Vigilância Sanitária. Brasília- DF. 2014. Disponível em: http://bvsms.saude.gov.br/bvs/publicacoes/documento_referencia_programa_nacional_se guranca.pdf

9. Tase TH, Lourenção DCA, Bianchini SM, Tronchin DMR. Identificação do paciente nas organizações de saúde: uma reflexão emergente. Rev Gaúcha Enferm. 2013;34(2):196-200. DOI: http://dx.doi.org/10.1590/S198314472013000300025

10. Hoffmeister LV, Moura GMSS. Uso de pulseras de identificación en pacientes internados en un hospital universitário. Rev. Latino-Am. Enfermagem ene.-feb. 2015. 23(1):36-43. DOI: 10.1590/0104-1169.0144.2522.

11. Tres DP, Oliveira JLC, Vituri DW, Alves SR, Rigo DFH, Nicola AL. Qualidade da assistência e segurança do paciente: avaliação por indicadores. Cogitare Enferm. 2016 2101-08. DOI: http://dx.doi.org/10.5380/ce.v21i5.44938

12. Porto TP, Rocha PK, Lessmann JC, Souza S, Kretzer L, Anders JC. Identificação do paciente em unidade pediátrica: uma questão de segurança. Revista Sociedade Brasileira de Enfermagem Pediátrica. 2011. 11(2).67-74. Disponível em: http://sobep.org.br/revista/component/zine/article/142-identificao-dopaciente-em-unidade-peditrica-uma-questo-de-segurana.html.

13. Suñol R, et al. Implementation of patient safety strategies in European hospitals. Qual Saf Health Care. 2009;18 Suppl1:57-61. DOI: http://dx.doi.org/10.1136/qshc.2008.029413.

14. Tase TH, Tronchin DMR. Sistemas de identificação de pacientes em unidades obstétricas e a conformidade das pulseiras. Acta paul. enferm. 2015. 28(4). DOI: http://dx.doi.org/10.1590/1982-0194201500063. 
15. Smith AF, Casey K, Wilson J, Fischbacher-Smith D. Wristbands as aids to reduce misidentification: an ethnographically guided task analysis. Int J Qual Health Care. 2011. 23. 590-599. DOI: 10.1093/intqhc/mzr045.

16. Macedo MCS, Almeida LF; Assad LG, Rocha RG, Rangel Ribeiro GSR, Vasconcelos Pereira, LMV. Identificação do paciente por pulseira eletrônica numa unidade de terapia intensiva geral adulta. Revista de Enfermagem Referência. 2017. 13. 63-70. DOI: http://dx.doi.org/10.12707/RIV16087.

17. Gomes APTS, Querido DL, Silva GRG, Almeida LFA, Rocha RG. Identificação do paciente em neonatologia para assistência segura. Cogitare Enferm. 2017. (22)3: e49501. DOI: http://dx.doi.org/10.5380/ce.v22i3.49501.

18. Ventura CMU, Alvez JGB, Meneses JA. Eventos adversos em Unidade de Terapia Intensiva Neonatal. Rev. bras. enferm. 2012. 65(1):49-55. DOI: http://dx.doi.org/10.1590/S0034-71672012000100007.

19. Teixeira TCA, Cassiani SHB. Análise de causa raiz: avaliação de erros de medicação em um Hospital Universitário. Rev. Esc. Enferm. USP. 2010. 44(1):139-46. DOI: http://dx.doi.org/10.1590/S0080-62342010000100020

Recebido: 12 de dezembro de 2017. Publicado: 19 de janeiro de 2018

Correspondência: Camila Girardi. E-mail: camilagirardi.vel@hotmail.com

Conflito de Interesses: os autores declararam não haver conflito de interesses.

(C) This is an Open Access article distributed under the terms of the Creative Commons Attribution License, which permits unrestricted use, distribution, and reproduction in any medium, provided the original work is properly cited 